\title{
Naked foraminiferans revealed
}

t is generally assumed that the first fossil appearance of a group of organisms corresponds to its evolutionary origin. But we have molecular evidence that extant members of the most abundant microfossilforming group, the Foraminifera, include 'naked' amoeboid species, indicating that ancestral foraminiferans could be unfossilized. This means that the origin of the group might be much earlier than has been deduced from the fossil record. This might help to explain the conflicting molecular and fossil data on the origin of the Foraminifera.

Foraminifera are classically defined as marine, shelled protists. Although the foraminiferan fossil record extends to the Cambrian period ${ }^{1}$, molecular data ${ }^{2}$ indicate a much earlier origin for these important microfossils. This disparity suggests that microfossils do not provide adequate evidence for the origin of this group. Such a gap in the fossil record could be explained by the existence of non-skeletonizing ('naked') foraminiferans that were not preserved in sediments. However, as foraminiferans did not include a naked species, we looked for the potential foraminiferan ancestor within the class Athalamea, a sister group to the Foraminifera ${ }^{3}$.
We have obtained ribosomal DNA and actin gene sequences from Reticulomyxa filosa, a well-known "giant freshwater amoeba" ${ }^{4}$ that lacks a shell but possesses pseudopodia that are strikingly similar to those of foraminiferans ${ }^{5}$. Phylogenetic analyses of these molecular data show that $R$. filosa is actually a foraminiferan, indicating that certain foraminiferans might lack a shell and live in freshwater environments.

The complete small subunit (SSU) rDNA sequences were obtained from $R$. filosa and five foraminiferan species. The SSU rDNA of $R$. filosa shows several common features with other foraminiferans, including a very low $\mathrm{G}+\mathrm{C}$ content $(32.6 \%)$ and several foraminiferan-specific insertions located in the conserved regions of the gene that make it unusually long $(3,347$ nucleotides). Phylogenetic analyses of SSU rDNA sequences, using different computational methods, consistently show that Reticulomyxa branches within the Foraminifera (Fig. 1a). Its position within the Foraminifera is not well established, but a comparison of more than 100 foraminiferan partial SSU rDNA sequences (not shown) suggests that the genus is most closely related to some primitive thecate Allogromiida.

These results were confirmed by an

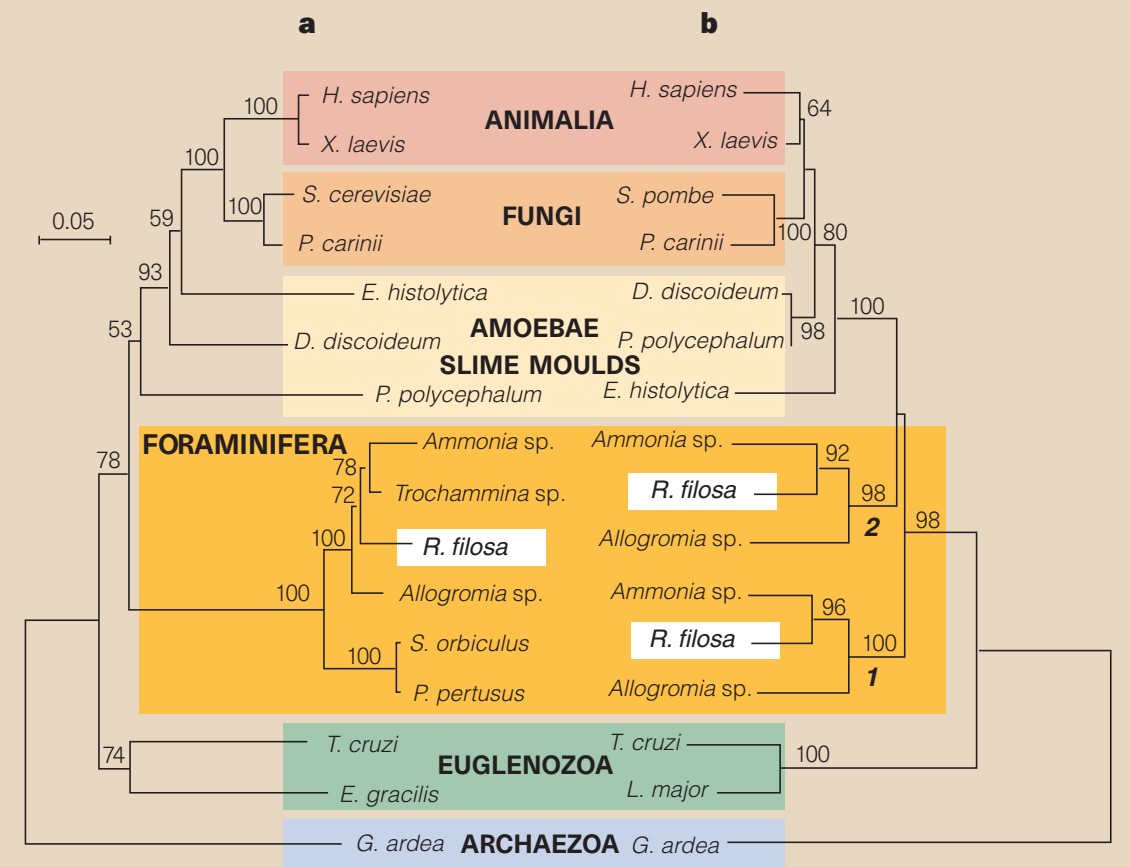

Figure 1 Phylogenetic position of Reticulomyxa filosa and foraminiferans. The position was inferred from complete sequences of SSU rDNA (a) and actin amino acids (b), using the neighbour-joining method with Kimura 2 and Dayhoff's PAM (Percent Accepted Mutations) matrix distances, respectively. The two foraminiferan actin gene families are numbered 1 and 2 . The scale bar corresponds to the number of substitutions per site. The GenBank accession numbers of new sequences reported here are AJ 132367 to AJ 132375. analysis of foraminiferan actin-coding genes. Two actin gene families were found in Reticulomyxa as well as in the two foraminiferans examined (Allogromia sp. and Ammonia sp.). Both actin gene sequences branch next to the Euglenozoa in the lower part of the phylogenetic tree (Fig. 1b) in a position remarkably similar to that suggested by analysis of the SSU rDNA data. Each actin family forms a clade supported by a high bootstrap value $(98 \%$ and $100 \%$ ) in which Allogromia appears as a sister group to Reticulomyxa and Ammonia. These relationships are supported by high bootstrap values $(92 \%$ and $96 \%)$ and remain stable in all analyses based on amino acid and DNA sequences.

The placement of Reticulomyxa within the Foraminifera leads us to challenge the micropalaeontological bias imparted to this group: the Foraminifera can no longer be defined strictly as shelled protists. Indeed, their only taxonomically consistent morphological character is the presence of granuloreticulopodia. Although $R$. filosa probably lost its shell secondarily as an adaptation to the freshwater environment, our findings nevertheless suggest that ancestral foraminiferans could have been naked and existed well before the dawn of skeletonization. This hypothesis helps to reconcile the conflicting molecular and fossil data on the origin of the Foraminifera. Our data also provide support for recent molecular studies ${ }^{6,7}$ that implicate a prolonged period of Precambrian diversification among the major eukaryotic groups.

We predict that naked foraminiferans similar to their hypothetical ancestors might still be living in the marine environment. Finding these species is essential for future molecular studies of the group. Jan Pawlowski ${ }^{\star} \dagger$, Ignacio Bolivar ${ }^{*}$, José Fahrni ${ }^{\star}$, Colomban de Vargas ${ }^{\star}$, Samuel S. Bowser

${ }^{*}$ Department of Zoology and Animal Biology, University of Geneva,

1224 Chêne-Bougeries, Geneva, Switzerland e-mail: pawlow@sc2a.unige.ch $\dagger$ Museum of Natural History, 1211 Geneva 4, Switzerland

$\ddagger$ Wadsworth Center,

New York State Department of Health, P.O. Box 509, Albany, New York 12201, USA

\footnotetext{
1. Culver, S. J. Science 254, 689-691 (1991).

2. Pawlowski, J. et al. Mol. Biol. Evol. 14, 498-505 (1997).

3. Lee J. J., Hutner, S. H. \& Bovee, E. C. (eds) An Illustrated Guide to the Protozoa (Allen, Lawrence, Kansas, 1985).

4. Koonce, M. P., Euteneuer, U. \& Schliwa, M. J. Cell Sci. (suppl. 5) 145-159 (1986).

5. Travis, J. L. \& Bowser, S. S. in Biology of Foraminifera (eds Lee, J. J. \& Anderson, O. R.) 91-156 (Academic, London, 1991). 6. Cooper, A. \& Fortey, R. Trends Ecol. Evol. 13, 151-156 (1998).

7. Bromham, L., Rambaut, A., Fortey, R., Cooper, A. \& Penny, D. Proc. Natl Acad. Sci. USA 95, 12386-12389 (1998).
} 